\title{
Generation of input signals for ArF amplifiers
}

\author{
S. Szatmári* and F. P. Schäfer \\ Abteilung Laserphysik, Max-Planck-Institut für biophysikalische Chemie, Postfach 2841, D-3400 Göttingen, \\ Federal Republic of Germany
}

Received April 3, 1989; accepted June 12, 1989

\begin{abstract}
Four different schemes for the generation of femtosecond input pulses for ArF amplifiers are described. Pulse energies of $100 \mathrm{~nJ}$ were obtained with phase-matched frequency mixing of a Raman-shifted frequency-doubled pulse derived from a 537-nm femtosecond dye-laser pulse. Excellent spatial, spectral, and amplitude stability was obtained when a broadband, nanosecond dye laser at $690 \mathrm{~nm}$ was used as a Raman seed pulse. Preliminary amplification experiments resulted in 0.5-mJ ArF output pulses of 340 fsec.
\end{abstract}

\section{INTRODUCTION}

Short-pulse excimer lasers are important tools for studies of multiphoton processes. ${ }^{1}$ It is known that $\mathrm{KrF}$ is superior to $\mathrm{XeCl}$ (Ref. 2) and $\mathrm{XeF}$ (Ref. 3) operated as a short-pulse amplifier, permitting efficient amplification of $60-\mathrm{fsec}$ pulses. ${ }^{4}$ ArF is of even greater interest than $\mathrm{KrF}$ owing to its shorter wavelength (193 nm versus $248 \mathrm{~nm}$ for $\mathrm{KrF}$ ). Its bandwidth should permit the amplification of nearly as short pulses as for $\mathrm{KrF}^{5}$ and the energy-extraction efficiency is expected to be similar to that of $\mathrm{KrF}$ owing to the similarly repulsive ground state. ${ }^{1,2,6}$ However, efficient generation of subpicosecond seed pulses at $193 \mathrm{~nm}$ is difficult because of the lack of a suitable frequency-converting crystal and the increased difficulties related to the generation of subharmonics at shorter wavelengths with the commonly used techniques. Because of the above-mentioned difficulty, the only short-pulse amplification experiment at the ArF wavelength of which we are aware is the one reported in Ref. 7. In that experiment 1-mJ, 6-psec pulses at $580 \mathrm{~nm}$ were obtained by amplification of pulses from a synchronously pumped, mode-locked dye laser. These pulses were then frequency tripled in a strontium heat pipe, where $\sim 2-n J$ pulses at $193 \mathrm{~nm}$ were produced with a strong broadband background. By subsequent amplification of these pulses in a four-stage ArF amplifier, 10-psec, 30-mJ pulses were obtained. Owing to the necessary high amplification, the amplified spontaneous emission (ASE) was also high, even when spectral and spatial filtering were used. When the energy of the short pulse was increased from 5 to $30 \mathrm{~mJ}$, the ASE background increased, even from 5 to $200 \mathrm{~mJ}$.

The extension of amplification at $193 \mathrm{~nm}$ to the subpicosecond time scale is straightforward, especially when the generation of short seed pulses can be done with improved efficiency.

Since our distributed-feedback dye-laser- (DFDL) based femtosecond dye-laser system ${ }^{4}$ can in principle generate subpicosecond pulses at any wavelength between $\sim 380 \mathrm{~nm}$ and $1.8 \mu \mathrm{m}, 8,9$ the lack of a suitable frequency-doubling crystal forced us to overcome the problem of seed-pulse generation at $193 \mathrm{~nm}$ by different frequency-conversion schemes. In this paper we present the results of these approaches.

\section{EXPERIMENTAL REALIZATIONS}

Several different frequency-conversion schemes were tested, using the output of an excimer-laser-pumped subpicosecond dye laser similar to those that were used in the $\mathrm{XeCl},{ }^{10} \mathrm{KrF},{ }^{4}$ and $\mathrm{XeF}$ (Ref. 3) amplification experiments. A schematic of this laser system is shown as Fig. 1. One channel of a double-discharge excimer laser (EMG 150, Lambda-Phys$\mathrm{ik}$ ), delivering $80-\mathrm{mJ}, 15-\mathrm{nsec}$ pulses at $308 \mathrm{~nm}$, is used to pump the dye-laser system. It consists of an intermediate dye laser, ${ }^{9}$ the DFDL master oscillator, ${ }^{8}$ and a power amplifier. The intermediate dye laser is a cascade laser system, in which the combined pulse-forming effect of dye oscillators, saturated amplifiers, and gated saturable absorbers results in a single output pulse of $\sim 8-10$-psec duration at $365 \mathrm{~nm}$ (for details see Ref. 9). These pulses have an energy of $\sim 4$ $\mu \mathrm{J}$ and are used for pumping the DFDL master oscillator. The DFDL used here is the so-called microscope-objective DFDL, ${ }^{8}$ in which the interference fringes necessary for DFDL operation are created by imaging a coarse transmission grating onto the active medium by the use of a microscope objective (for further details see Ref. 8).

Since for the different frequency-conversion schemes different DFDL wavelengths were necessary, the easy tuning of the wavelength of this kind of DFDL was highly advantageous. The output of the DFDL was amplified in a two- or three-stage dye-amplifier chain pumped by the rest of the available excimer pump energy.

\section{Frequency Tripling}

The newly introduced $\beta-\mathrm{BaB}_{2} \mathrm{O}_{4}(\mathrm{BBO})$ crystal $^{11}$ has been reported to be transparent down to $189 \mathrm{~nm}$ (Ref. 11) and phase matchable for second-harmonic generation (SHG) to $204.8 \mathrm{~nm} .{ }^{12}$ The shortest wavelength that can be reached through third-harmonic generation (THG) by mixing a fundamental wavelength with its second harmonic is 197.3 nm. ${ }^{13}$

The efficiency of frequency conversion in BBO is poor when one is working close to the short-wavelength limit (i.e., close to $90^{\circ}$ crystal orientation) because of the strong angular dependence of the effective nonlinear-optical constant. ${ }^{12}$ This sudden loss of efficiency near $90^{\circ}$ phase-matching an- 


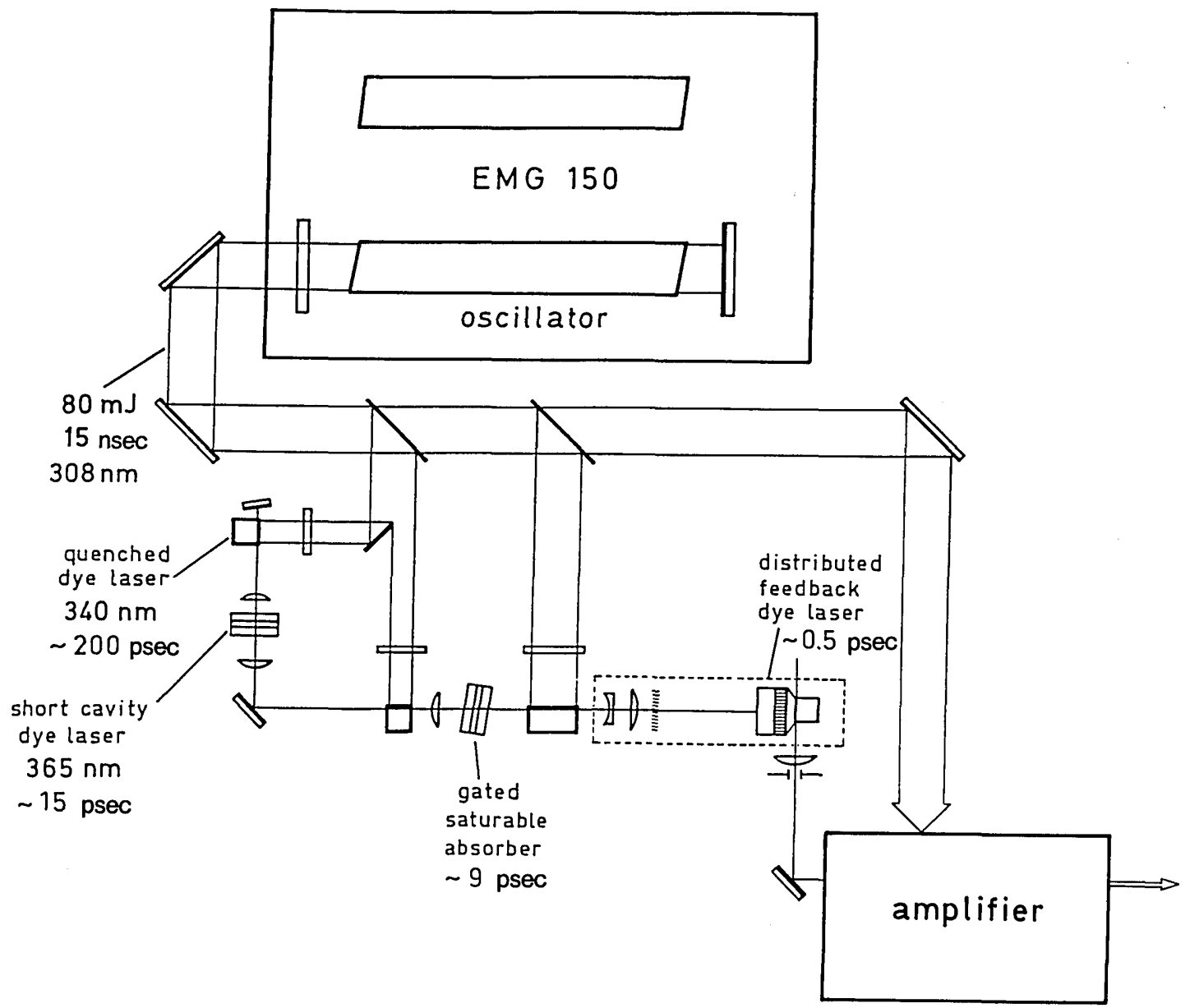

Fig. 1. Schematic of the excimer-laser-pumped subpicosecond dye-laser system.

gle has been noted in both SHG (Ref. 12) and THG (Ref. 13) experiments.

When the crystal is cooled to $\sim 100 \mathrm{~K}$, the shortest wavelength for SHG and THG is 203.4 (Ref. 14) and $195.3 \mathrm{~nm},{ }^{14,15}$ respectively. Even when this cooled crystal is used, the short-wavelength limit of THG is still far from the tuning range of $\mathrm{ArF}$, which is centered at $193.3 \mathrm{~nm}$ and already ceases at $194.3 \mathrm{~nm} .^{5}$

On the other hand, when short pulses and correspondingly thin crystals are used, the wavelength dependence of efficiency for a given crystal orientation is expected to decrease. Therefore one might expect some slowly decreasing signal even beyond the short-wavelength limit, which can reach the ArF gain band.

For this reason we have measured the wavelength dependence of the efficiency of mixing a fundamental wavelength from 580 to $595 \mathrm{~nm}$ with its second harmonic as shown in Fig. 2. The pulses at the fundamental wavelength were generated by the system shown in Fig. 1. After amplification, typically $50-\mu \mathrm{J}$ pulses of $\sim 500$-fsec duration were obtained.

The output beam was linearly polarized with a vertical $E$ vector, which was then focused by an $f=200-\mathrm{mm}$ lens and frequency doubled by a $0.2-\mathrm{mm}$-thick, $42^{\circ}$-cut BBO crystal positioned before the focus of the lens. The mixing crystal was $0.2-\mathrm{mm}, 90^{\circ}$-cut $\mathrm{BBO}$, positioned just at the focus. Since the two wavelengths to be frequency mixed had crossed polarizations, the second $\mathrm{BBO}$ crystal was turned by $45^{\circ}$ around the axis of the beam. The energy of the third harmonic, measured by a calibrated Hamamatsu R 1081 solar-blind photomultiplier, as a function of wavelength is shown in Fig. 2. One observes a sharp drop in efficiency at wavelengths longer than the cutoff wavelength, in agreement with the observations of Ref. 13. However, the THG energy does not drop to zero, even when the wavelength is decreased beyond the cutoff wavelength (non-phasematched frequency mixing) but stays at $\sim 8 \mathrm{~nJ}$. It is seen from the figure that the effect of phase matching is negligible

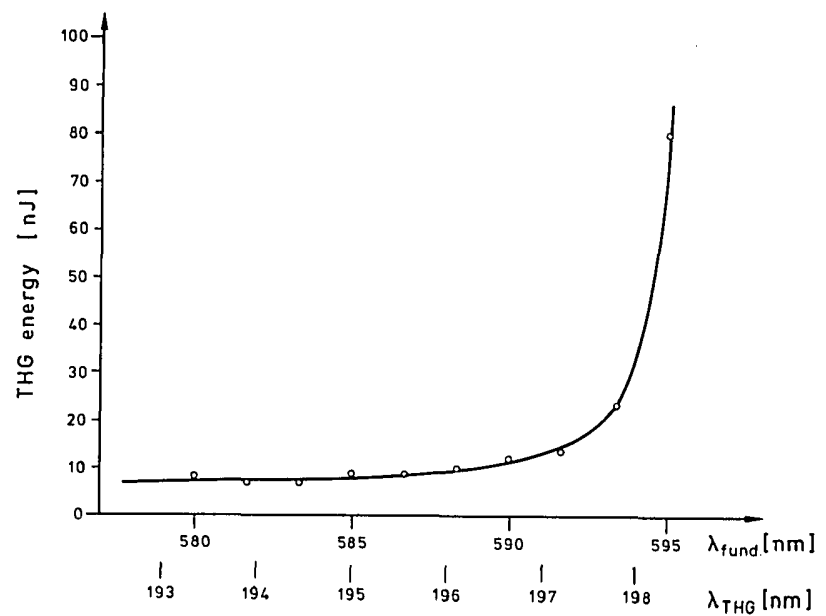

Fig. 2. Wavelength dependence of the third harmonic generated by a 0.2 -mm-thick, $90^{\circ}$-cut $\mathrm{BBO}$ crystal. 
at the desired 193.3-nm wavelength in spite of the relatively small separation of this wavelength and the cutoff wavelength $(197.3 \mathrm{~nm})$.

This experiment was repeated with a cooled BBO crystal (at $\sim 100 \mathrm{~K}$ ). One could observe the expected shift of the cutoff wavelength, ${ }^{15}$ but no better result was achieved at 193 nm.

This means that in the cases of both THG and SHG one has to work in a purely non-phase-matched condition, which makes SHG preferable.

\section{Frequency Doubling}

For the non-phase-matched SHG experiments the wavelength of the dye laser was set to $386.6 \mathrm{~nm}$. The typical output energy was $\sim 30 \mu \mathrm{J}$, which was then focused by an $f=$ $200 \mathrm{~mm}$ lens onto different samples $\left(\mathrm{CaF}_{2}, \mathrm{LiF}, \mathrm{KDP}, \mathrm{BBO}\right.$, ADP). The samples were illuminated at $0^{\circ}$ angle of incidence and were rotated around the axis of the beam to get maximum signal. The best results are obtained for the ADP crystal, for which $12-\mathrm{nJ}$ pulses at $193 \mathrm{~nm}$ were generated. This method is simple; however, here the energy of the SHG is limited to the $\sim 10-\mathrm{nJ}$ range.

The two examples discussed above showed that, when one is using non-phase-matched frequency conversion, the achievable output signal barely exceeds the $10-\mathrm{nJ}$ range for our input energies. To have efficient frequency conversion below the $198 \mathrm{~nm}$ limit one has to utilize other frequencyconversion schemes (e.g., anti-Stokes stimulated Raman scattering) or to use two different wavelengths to be mixed in the BBO crystal, for which the phase-matching condition can be fulfilled even beyond $193 \mathrm{~nm}$, as demonstrated in Ref. 16. However, for the generation of these wavelengths either two different oscillators or (preferably with only a single oscillator) other frequency-conversion schemes are necessary.

\section{Frequency Conversion Including Raman Oscillators}

Both Stokes and anti-Stokes stimulated Raman scattering are well developed and widely used techniques for converting radiation to longer or shorter wavelengths with high efficiency. ${ }^{17-24}$

Our first effort to reach the wavelength of $193 \mathrm{~nm}$ by using Raman conversion was the coherent anti-Stokes Raman conversion of $210-\mathrm{nm}$ radiation into $193 \mathrm{~nm}$, using hydrogen as a Raman scatterer; but this approach failed to work. In this experiment the initial wavelength of the Raman conversion $\left(\lambda_{0}=210 \mathrm{~nm}\right)$ was reached through frequency doubling in a BBO crystal. For this reason we had to use a Raman scatterer having enough shift (for hydrogen, $\Delta \bar{\nu}=4155 \mathrm{~cm}^{-1}$ and $\lambda_{0}=210 \mathrm{~nm}$ ) for which the initial wavelength can be reached through efficient frequency doubling in BBO. On this time scale hydrogen is a highly transient scatterer, ${ }^{24}$ for which the threshold of Raman conversion is only energy dependent. For the less transient scatterer $\mathrm{CH}_{4}$, lower threshold is expected, but the shift $\left(\Delta \bar{\nu}=2916 \mathrm{~cm}^{-1}\right)$ is relatively small, corresponding to an initial wavelength of $\lambda_{0}$ $=205 \mathrm{~nm}$, which can be reached only with poor efficiency by the use of SHG in BBO. ${ }^{12}$

Because of the energy dependence of the threshold of transient Raman scattering, other power-dependent processes (self-focusing, self-phase modulation, continuum generation) become easily competitive when one is converting subpicosecond pulses into the first Stokes line. The conditions are even worse when conversion into higher orders or into anti-Stokes lines is needed. We have found from the practical point of view that the only useful conversion of subpicosecond pulses with Raman oscillators is conversion into the first Stokes line.

In the next experiment with Raman conversion, we attempted to reach the 193-nm wavelength by frequency mixing of the Stokes-shifted Raman line and the second harmonic of the fundamental wavelength. This is actually a realization of the idea of extending the phase-matching condition for BBO to shorter wavelengths by mixing two different initial wavelengths. ${ }^{16}$ However, in this approach these two wavelengths are generated from a common source by downfrequency and upfrequency conversion.

It is known ${ }^{12,13}$ that one has to operate with a BBO crystal at phase-matching angles smaller than $\sim 83^{\circ}$ in order to get efficient frequency conversion. This corresponds to a wavelength that is longer than the cutoff wavelength by roughly 1 $\mathrm{nm}$. This means that the two wavelengths have to be chosen so that the phase-matching condition is fulfilled even for 192 $\mathrm{nm}$. In spite of the slight discrepancy in the calculated phase-matching conditions obtained from the dispersion formulas of Chen ${ }^{11}$ and Kato, ${ }^{12}$ a good estimate of the two necessary wavelengths can be made by using the experimental data of Ref. 16 and comparing those with the dispersion formulas. ${ }^{25}$ We concluded that one needs a shift of more than $\sim 4000 \mathrm{~cm}^{-1}$, which can be reached only by vibrational Raman scattering in hydrogen or by electronic Raman scattering. When hydrogen is used as a Raman scatterer, the fundamental wavelength must be set to $537 \mathrm{~nm}$, giving a second-harmonic wavelength of $268.5 \mathrm{~nm}$ and a first-Stokesshifted line at $691 \mathrm{~nm}$. This results in a sum frequency at $193.3 \mathrm{~nm}$, for which the efficiency of sum-frequency conversion is expected still to be high in BBO. By optimizing our femtosecond dye-laser system to the highest output energy, $\sim 200-\mu \mathrm{J}$ pulses of $\sim 500$-fsec duration are obtained at 537 $\mathrm{nm}$.

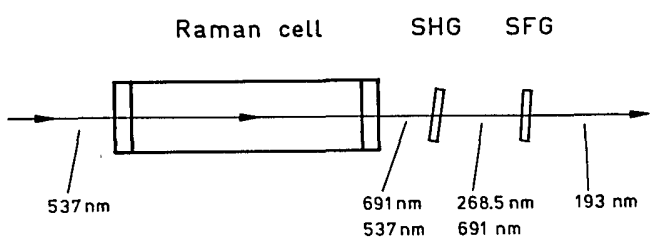

(a)

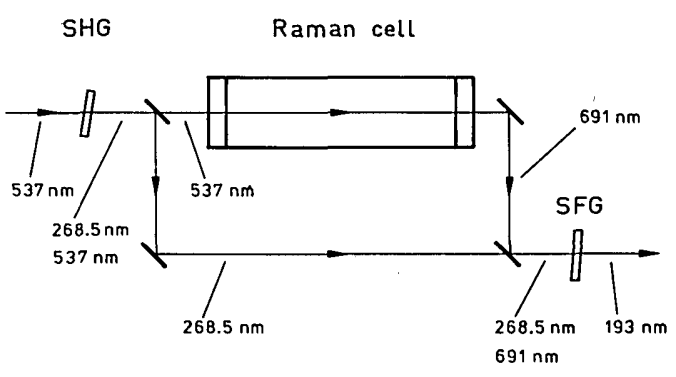

(b)

Fig. 3. Possible schemes for frequency conversion including a Raman oscillator. 
Possible schemes for frequency conversion are shown in Fig. 3. Figure 3(a) shows the simplest possible in-line arrangement, in which the 537-nm fundamental wavelength is first converted to $691 \mathrm{~nm}$ by a Raman shift in hydrogen and then the rest of the fundamental is used for frequency doubling. Then the 268.5- and 691-mm wavelengths are mixed in the following sum-frequency-generation (SFG) crystal. (In the figure, at each point of the system only those wavelengths are indicated that are important from the point of view of frequency conversion.) It is known from the theory of transient Raman generation ${ }^{26}$ that the first Stokes pulse follows the pump pulse with a delay $\left(t_{d}\right)$ comparable with the duration of the pump pulse $\left(t_{p}\right)\left(t_{d} \approx 0.9 t_{p}\right)$. Owing to the group-velocity dispersion of the window material of the $\mathrm{Ra}$ man cell, this delay is decreased, and by proper choice of its thickness complete temporal overlap can be achieved. This synchronism can be maintained in the later stages by the use of thin crystals in the positions of SHG and SFG.

One problem to be considered is the orthogonal polarization of the second harmonic and the Raman pulse, which can be overcome, albeit at the expense of efficiency, by rotating the optical axis of the SFG crystal by $45^{\circ}$ around the axis of the beam. A better solution is to rotate selectively the polarization of one of the beams by a dichroic half-wave plate, while preserving the state of the polarization for the other (see below).

We found that the main problem in using the scheme in Fig. 3(a) is the spatial and spectral distortion of the fundamental pulse during Raman conversion. Even in the optimum conditions, when the $200-\mu \mathrm{J}$ output pulse of the dye laser was gently focused onto the Raman cell containing $\mathbf{4 5}$ bars of hydrogen and positioned $\sim 1 \mathrm{~m}$ beyond the last amplifier, strong self-phase modulation and self-focusing occurred at the Raman threshold. The spatial distortion made the SHG process ineffective, and the spectral distortion of the fundamental was then converted to both the Raman and the second-harmonic spectra, appearing as statistically fluctuating lines.

These difficulties could partly be avoided if SHG could be done before the Raman process. However, this cannot happen when an in-line arrangement is used, owing to the group- velocity dispersion of the cell windows. Figure $3(\mathrm{~b})$ shows a solution for this case, in which the fundamental and the second harmonic are separated after SHG by a dichroic mirror. Then the rest of the fundamental is used for the generation of the first Stokes line, which is then recombined with the second-harmonic by using another dichroic mirror, in a way to ensure exact synchronism for SFG.

When this arrangement is used, spectral distortion is expected to be present only in the Raman spectrum. In order to minimize this distortion and to increase the effective iteraction length, we should use the Raman cell in a double-pass configuration. For this reason and because of the lack of suitable dichroic mirrors, the arrangement finally used in our experiments was a modified version of Fig. 3(b), as shown in Fig. 4.

The output of the dye laser was gently focused onto the 50 cm-long Raman cell containing 45 bars of hydrogen, positioned $\sim 1 \mathrm{~m}$ after the last amplifier. SHG is done just after the last amplifier by using a 0.2 -mm-thick, $52^{\circ}$-cut $\mathrm{BBO}$ crystal. It is positioned in a special holder consisting of two $\varphi=10^{\circ}$ wedged plates oriented oppositely. This holder is filled with an immersion liquid. Then the fundamental passes through this construction as if it were a plane-parallel plate, but the second harmonic generated between the two wedged plates-owing to the different refractive indices of the wedges for these two wavelengths-deviates slightly (by $\sim 0.5^{\circ}$ ). This angular separation is just enough to separate the second harmonic (shown as the dashed lines in Fig. 4) and to send it via mirror $M_{1}$ through a delay line in order to compensate for the delay of the other arm where the Raman conversion is done. After passing through the Raman cell, the main pulse together with the Raman pulse is sent back to the cell by an $R=500-\mathrm{mm}$ concave aluminum mirror. In the second pass, the cell is used as a Raman amplifier. ${ }^{23,27,28}$ The synchronization between the pump and Raman pulses is readjusted by proper choice of the thickness of the cell window adjacent to the mirror. The thickness optimum is $\sim 10 \mathrm{~mm}$ of quartz glass.

The beam after the second pass-which has a small $\left(\sim 0.3^{\circ}\right)$ angle to the incoming beam-is directed to lens $L$ via mirror $\mathbf{M}_{2}$. The curvature of mirror $\mathbf{M}_{4}$, which directs the

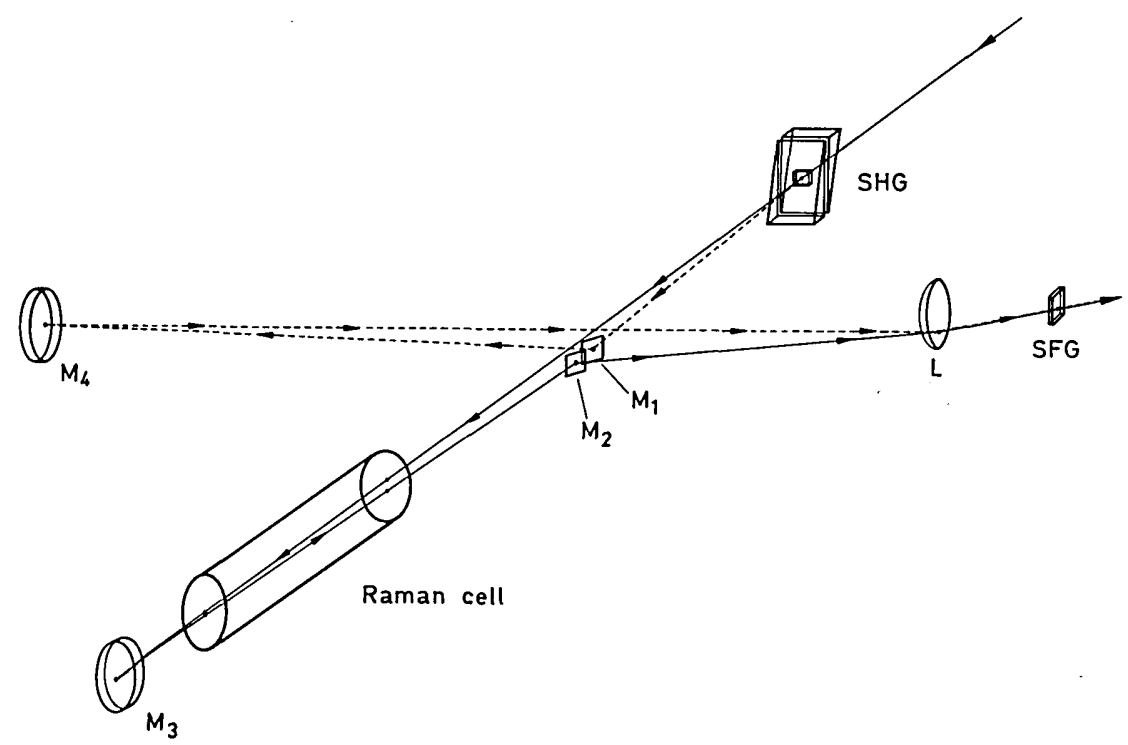

Fig. 4. Experimental realization of frequency conversion including a Raman oscillator. 


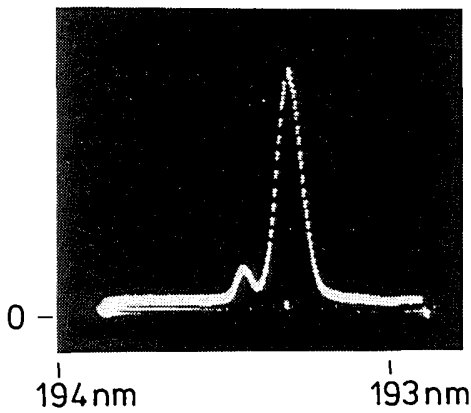

(a)

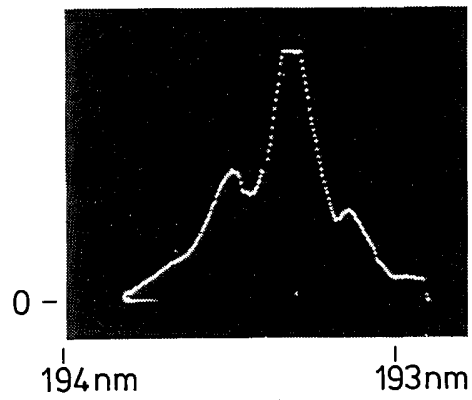

(b)

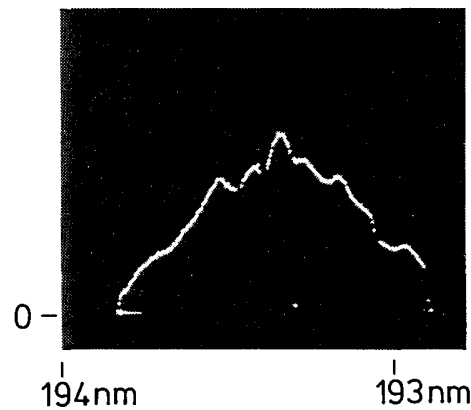

(c)

Fig. 5. Spectrum of the output pulse at $193 \mathrm{~nm}$ using (a) 1-mm and (b) 0.2-mm SFG crystals. (c) Spectrum of the ASE of the ArF amplifier.

second harmonic toward $\mathrm{L}$, is chosen so that the beam diameters, both for the second harmonic and for the Raman beam, are the same at the position of L. By proper adjustment and translation of $\mathrm{M}_{4}$, the two beams are made spatially and temporally overlapping; however, they enclose an angle of $\sim 0.5^{\circ}$. By using an off-axis area of the focusing lens, owing to the dispersion of the lens the two beams can be set parallel and have their foci close to each other. The SFG crystal-a $90^{\circ}$-cut $\mathrm{BBO}$ crystal-is positioned between the two focal points. During Raman generation both the originally linearly polarized pump and the Raman beam became unpolarized. Then the horizontal component was mixed by the SFG crystal with the horizontally polarized second harmonic. The energy of the mixed frequency at $193 \mathrm{~nm}$ was $\sim 80 \mathrm{~nJ}$.

This beam could easily be separated from the 268.5- and 691-nm radiation by the use of a pair of dichroic mirrors and appeared as an easily visible circular spot when a white paper was inserted as a fluorescent screen into the beam. The spectrum of the 193-nm pulse is shown in Figs. 5(a) and 5 (b) for 1 - and $0.2-\mathrm{mm}, 90^{\circ}$-cut BBO crystals, respectively, used for SFG. The spectral narrowing due to the limited bandwidth of the long crystal is clearly seen by comparing the two spectra. In Fig. 5(a) the narrow line could be tuned over the gain spectrum of the ArF [shown in Fig. 5(c) for comparison] by rotating the SFG crystal. From these figures one can conclude that even the shorter $(0.2-\mathrm{mm})$ crystal is somewhat too long for this application. The line structure of the spectrum in Fig. 5(b), in which both the relative amplitude and the position of these lines was statistically fluctuating, is the result of the spectral distortion that occurs during Raman conversion.

Preliminary amplification experiments were performed that used these pulses as input pulses for the second discharge channel of an EMG 150 pump laser filled with 7.5 mbars of fluorine, 350 mbars of argon, and with helium to a total pressure of 2.5 bars. In a double-pass arrangement (similar to that used in Ref. 4) the energy was boosted to $\sim 0.5 \mathrm{~mJ}$ with less than $1 \%$ ASE content. The beam diameters for the first and second passes were $\sim 1$ and $4 \mathrm{~mm}$, respectively. In the second pass the maximum beam diameter was limited by the relatively narrow discharge. The pulse duration of the output was measured by the twophoton ionization autocorrelator technique, using $\mathrm{NO}$ gas as in Ref. 4. (A detailed description of the autocorrelator is given in Ref. 29.) Two autocorrelation curves are shown in Fig. 6, where the curve of Fig. 6(a) is measured directly after the output, and that of Fig. 6(b) after compression in a single-pass, double-prism pulse compressor consisting of two $60^{\circ} \mathrm{LiF}$ prisms separated by $2.3 \mathrm{~m}$. The autocorrelation width is 1.3 psec for the uncompressed pulse, decreasing to 530 fsec after compression, corresponding to 340 -fsec pulse duration assuming $\operatorname{sech}^{2}$ pulse shape.

By studying the spectral and temporal behavior of the short pulses shown in Figs. 5 and 6, one sees that the main drawback of this frequency-conversion scheme (besides its

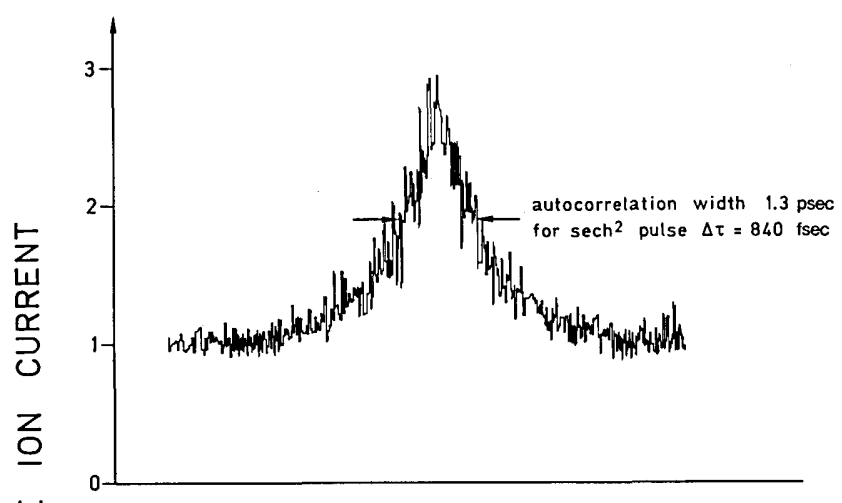

(a)

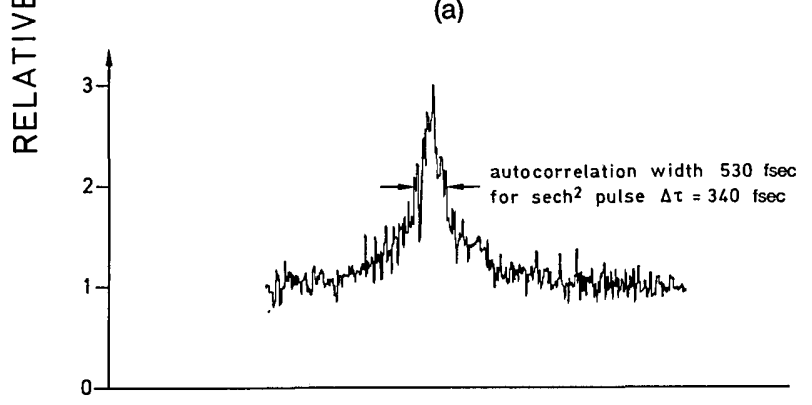

(b)

Fig. 6. Autocorrelation trace of the amplified 193-nm pulses (a) just after amplification and (b) with pulse compression. 
relative complexity) is the output modulated spectrum, which leads to a relatively long pulse duration. Even after pulse compression the pulse duration is roughly three times the Fourier limit of the ArF amplifier, and inherent uncompressable temporal wings are seen to be present on the autocorrelation curves.

In the above arrangement the Raman cell was used in the second pass as an amplifier, decreasing the focusing requirements and therefore the spectral distortions in the first pass. However, one had to achieve oscillation in the first pass, which already led to some spectral distortion of the fundamental. The main problem is that this spectrally distorted fundamental is then used as a pump pulse in the second pass, so that the gain spectrum of this Raman amplifier becomes distorted, resulting in an even more distorted output spectrum. It would be desirable to use the Raman cell only as an amplifier, which could lead to less required gain and therefore less distortion. However, in this case a suitable seed pulse is needed at the Raman wavelength.

\section{Frequency Conversion Using Raman Amplification}

It has been shown theoretically ${ }^{23}$ and also experimental$\mathrm{ly}^{23,27,28}$ that the spectral and spatial quality of a Raman beam and the efficiency of energy conversion into the first Stokes line can be improved significantly by injecting a properly synchronized input pulse at the desired Raman wavelength into a Raman cell, which then is used as a Raman amplifier. In all three of the references cited above the seed pulses were generated in another Raman oscillator, pumped by a fraction of the pump pulse, and served as input pulses for the Raman amplifier after spatial and spectral filtering.

In our experimental conditions the available pump energy of $\sim 200 \mu \mathrm{J}$ is just enough for pumping one cell. On the other hand, the necessary spatial and spectral filtering of the Raman pulse and its synchronization to the pump pulse in the amplifier make this method fairly complicated for practical use. Since the gain at the oscillation threshold of Raman amplifiers is in the range of $\sim 10^{10,23}$ one has a chance to saturate these amplifiers even with input pulses having extremely small intensity in the temporal and spectral gain windows of the Raman amplifier. This is demonstrated by the following numerical example. Assuming a Raman amplifier to be pumped by a 200- $\mu \mathrm{J}, 0.5$-psec transform-limited pulse, and using the $\sim 100-\mu \mathrm{J}$ output of an excimer-laserpumped broadband dye laser having a duration of $\sim 10 \mathrm{nsec}$ and approximately five times broader bandwidth than that of the Raman amplifier, one needs a gain of only $\sim 10^{4}$ to. reach saturation. Comparing this value with the gain corresponding to the self-oscillation threshold, one can expect deeply saturated amplification at a pump rate, where no oscillation occurs. Saturation effects are already apparent in Raman amplification experiments, when the amplified Stokes energy is larger than $2 \%$ of the input pump energy. ${ }^{23}$ We found a practical optimum at $\sim 10 \%$ : Here the dependence of the amplified Stokes energy on both the pump and the seed Stokes energy is already small, but pump depletion still does not play an important role in modulating the pump pulse. In this way one can stabilize the Stokes energy without affecting the temporal profile of the transmitted pump pulse. The advantages of using long and broadband dyelaser pulses are that one need not take care of any synchronization and that the Raman line can be tuned over the spectrum of the dye-laser pulse simply by tuning the pump pulse.

The experimental realization shown in Fig. 7 includes the DFDL amplifier chain, the broadband seed-pulse oscillator, the Raman amplifier, and the crystals. The DFDL pulse, after amplification, is sent through a telescope $(M=1)$, including a spatial filter discriminating against ASE. This telescope is adjusted to get a convergent beam through the second, 1.5-mm-diameter Bethune-type amplifier, so that its focus is beyond the Raman cell approximately $1 \mathrm{~m}$ after the last amplifier, where the SHG and SFG crystals are positioned. Under these conditions the Raman cell is far from the self-oscillation threshold. The seed pulses for the Raman amplifier are generated in the broadband dye laser, pumped by $\sim 10 \%$ of the pump energy directed to the last amplifier. This laser consists of a backmirror of $100 \%$ reflectivity and a glass plate as an outcoupler and uses a $1.5 \times$ $10^{-3} M$ solution of Pyridin 1 dye in methanol as an active medium. This results in an oscillation centered just at 691

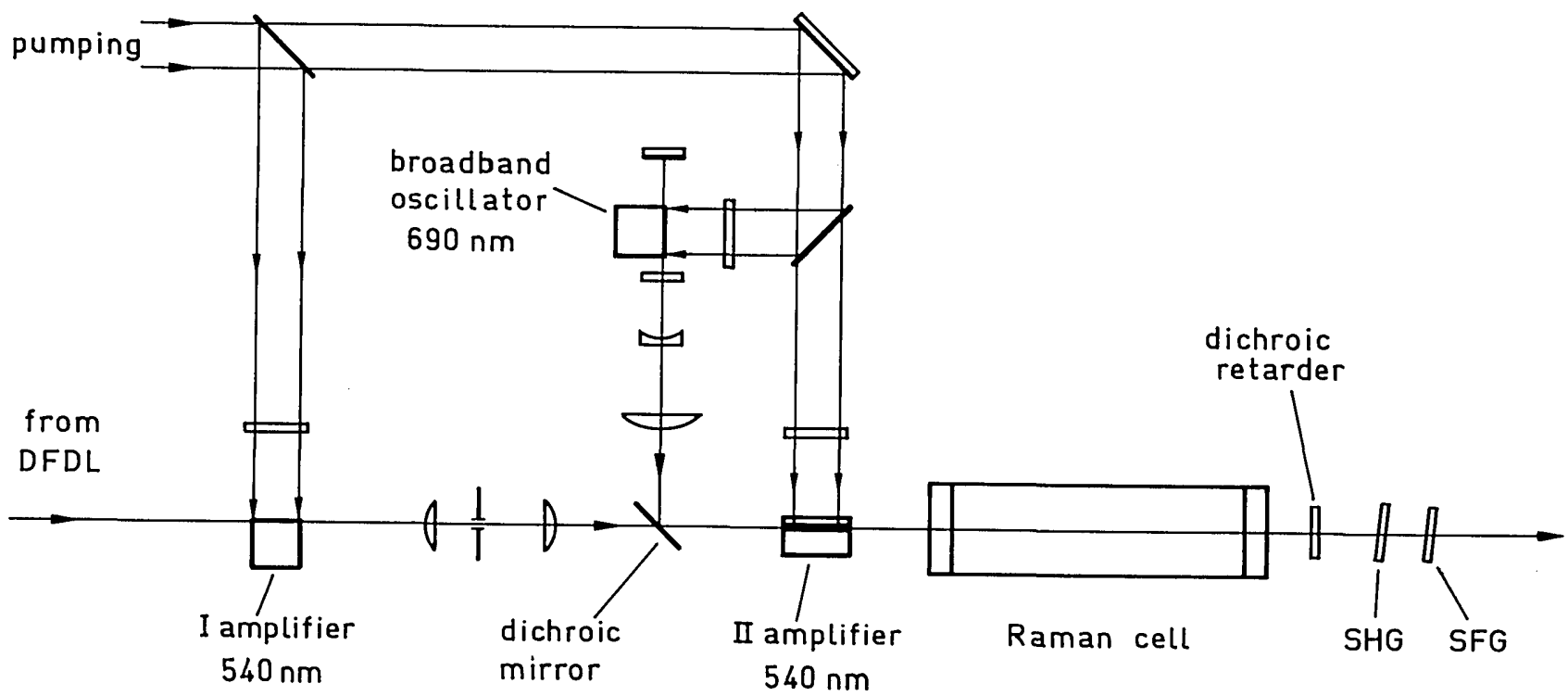

Fig. 7. Experimental realization of frequency conversion using Raman amplification. 
$\mathrm{nm}$, with a spectral width four to five times broader than needed for the Raman process. The output is then magnified in a Galilean telescope $(M=2)$ and combined with the $537-\mathrm{nm}$ pump pulse, using a dichroic mirror. (This is done before the last amplifier to minimize losses for the pump pulse.) By proper adjustment of the telescope and the beam-combining mirror the two beams are adjusted to be in exact spatial coincidence and to have similar divergence. Without the use of a polarizer, both beams are linearly polarized with a vertical $E$ vector. After Raman amplification the polarization of the fundamental is turned $90^{\circ}$ by a dichroic retarder while the polarization of the Raman pulse is left unchanged. This means that after frequency doubling of the fundamental in a $0.2-\mathrm{mm}, 52^{\circ}$-cut $\mathrm{BBO}$ crystal, the polarization of the second harmonic and the Raman pulse is the same, permitting efficient sum-frequency generation in the next $0.2-\mathrm{mm}$-thick, $90^{\circ}$-cut $\mathrm{BBO}$ crystal. With this arrangement $100-\mathrm{nJ}$ pulses at $193 \mathrm{~nm}$ were generated, exhibiting a broader spectrum than obtained with the earlier methods and free of any spectral modulation due to spectral distortion. Even the effect of the anticipated statistical spectral fluctuation of the broadband dye-laser oscillator on the final spectrum was eliminated by saturation of the gain during Raman amplification. The divergence of the output is comparable with that of the incoming pump and seed beams and is only slightly distorted by the imperfect optical quality of our thin BBO crystals. Research is in progress to use both crystals in a common container, filled with immersion liquid, and to use only antireflection-coated surfaces in order to optimize efficiency and to improve beam quality. We have also studied the dependence of the Raman and the SFG signals on the energy of both the pump and the seed pulses, and we found a broad range of strong saturation, resulting in stable operation. The pulse width obtainable with this arrangement and a BBO mixing crystal of 0.1-mm thickness (which was not available at the time of this writing) will be less than the $350 \mathrm{fsec}$, as measured with our 0.2$\mathrm{mm}$ BBO crystal and the third of the above arrangements. A detailed account of the results of amplification of the output pulse in $\mathrm{ArF}$ will be published elsewhere. ${ }^{30}$

\section{CONCLUSION}

We have studied four frequency-conversion schemes for generating seed pulses for ArF amplifiers at $193 \mathrm{~nm}$. With non-phase-matched SHG and THG in different crystals, we demonstrated a simple way to generate seed pulses with moderate $(\sim 10-n J)$ energy. The energy of the 193-nm pulse can be increased by roughly an order of magnitude if a $\mathrm{BBO}$ crystal is used for phase-matched SFG of signals obtained by SHG and Raman conversion of a fundamental frequency. Using this frequency-conversion scheme, an experimental arrangement is built, generating $80-\mathrm{nJ}$ pulses at $193 \mathrm{~nm}$. By double-pass amplification of these pulses in an ArF amplifier, 0.5-mJ, 850-fsec pulses are obtained with less than $1 \%$ ASE content. Additional pulse compression resulted in significant decrease of the pulse duration to $340 \mathrm{fsec}$.

If the Raman process involved is Raman oscillation, the output spectrum is always distorted, owing to nonlinear power-dependent processes that compete with the energydependent Raman generation. This problem is avoided by the use of Raman amplification. A simple in-line arrange- ment is developed that utilizes the Raman amplification of an excimer-laser-pumped dye-laser pulse. This arrangement is simple and reliable and can be built and operated without any synchronization requirement. With this arrangement the generation of $100-\mathrm{nJ}$ pulses at $193 \mathrm{~nm}$ is achieved with excellent spectral and spatial quality.

\section{ACKNOWLEDGMENTS}

The authors thank W. Mückenheim and I. N. Ross for useful advice. This research has been supported by the Bundesministerium für Forschung und Technologie and the Deutsche Forschungsgemeinschaft by the Gottfried-Wilhelm-Leibniz-Program.

* Permanent address, Research Group on Laser Physics of the Hungarian Academy of Sciences, József Attila Tudományegyetem University, Dóm tér 9, H-6720 Szeged, Hungary.

\section{REFERENCES AND NOTES}

1. C. K. Rhodes, ed., Excimer Lasers, 2nd ed., Vol. 30 of Topics in Applied Physics (Springer-Verlag, Berlin, 1984).

2. S. Szatmári and F. P. Schäfer, J. Opt. Soc. Am. B 4, 1943 (1989).

3. Q. Zhao, S. Szatmári, and F. P. Schäfer, Appl. Phys. B 47, 325 (1988).

4. S. Szatmári and F. P. Schäfer, Opt. Commun. 68, 196 (1988).

5. H. F. Döbele, M. Hörl, and M. Röwekamp, Appl. Phys. B 42, 67 (1987).

6. A. J. Taylor, R. B. Gibson, and J. P. Roberts, Appl. Phys. Lett. 52, 773 (1988).

7. H. Egger, T. S. Luk, K. Boyer, D. F. Muller, H. Pummer, T. Srinivasan, and C. K. Rhodes, Appl. Phys. Lett. 41, 1032 (1982).

8. S. Szatmári and F. P. Schäfer, Appl. Phys. B 46, 305 (1988).

9. S. Szatmári, Opt. Quantum Electron. 21, 55 (1989).

10. N. P. Ernsting, M. Kaschke, J. Kleinschmidt, K. H. Drexhage, and V. Huth, Chem. Phys. 122, 431 (1988).

11. C. Chen, B. Wu, A. Jiang, and G. You, Sci. Sin. 28, 235 (1985).

12. K. Kato, IEEE J. Quantum Electron. QE-22, 1013 (1986).

13. W. L. Glab and J. P. Hessler, Appl. Opt. 26, 3181 (1987).

14. P. Lokai, B. Burghardt, D. Basting, and W. Mückenheim, Laser Optoelektron. 3, 296 (1987).

15. P. Lokai, B. Burghardt, and W. Mückenheim, Appl. Phys. B 45, 245 (1988).

16. W. Mückenheim, P. Lokai, B. Burghardt, and D. Basting, Appl. Phys. B 45, 259 (1988).

17. T.R. Loree, R. C. Sze, and D. L. Barker, Appl. Phys. Lett. 31, 37 (1977).

18. V. Wilke and W. Schmidt, Appl. Phys. 16, 151 (1978).

19. V. Wilke and W. Schmidt, Appl. Phys. 18, 177 (1979).

20. T. R. Loree, R. C. Sze, D. L. Barker, and P. B. Scott, IEEE J. Quantum Electron. QE-15, 337 (1979).

21. D. J. Brink and D. Proch, Opt. Lett. 7, 494 (1982).

22. N. Morita, L. H. Lin, and T. Yajima, Appl. Phys. B 31, 63 (1983).

23. M. D. Duncan, R. Mahon, L. L. Tankersley, and J. Reintjes, J. Opt. Soc. Am. B 5, 37 (1987).

24. N. J. Everall, J. P. Partanen, J. R. M. Barr, and M. J. Shaw, Opt. Commun. 64, 393 (1987).

25. W. Mückenheim, Lambda Physik GmbH, Hans-BöcklerStrasse 10, Postfach 2663, D-3400 Göttingen, Federal Republic of Germany (personal communications).

26. R. L. Carman, F. Shimizu, C. S. Wang, and N. Bloembergen, Phys. Rev. A 2, 60 (1970).

27. M. J. Shaw, J. P. Partanen, Y. Owadano, I. N. Ross, E. Hodgson, C. B. Edwards, and F. O'Neill, J. Opt. Soc. Am. B 3, 1466 (1986).

28. N. J. Everall and E. Fujiwara, Rutherford Laboratory Report (1988), p. 124.

29. S. Szatmári, G. Kühnle, J. Jasny, and F. P. Schäfer, Appl. Phys. $\mathrm{B}$ (to be published). 